\title{
Hubbard model on triangular $N$-leg cylinders: Chiral and nonchiral spin liquids
}

\author{
Luca F. Tocchio $\odot,{ }^{1}$ Arianna Montorsi $\odot,{ }^{1}$ and Federico Becca ${ }^{2}$ \\ ${ }^{1}$ Institute for Condensed Matter Physics and Complex Systems, DISAT, Politecnico di Torino, I-10129 Torino, Italy \\ ${ }^{2}$ Dipartimento di Fisica, Università di Trieste, Strada Costiera 11, I-34151 Trieste, Italy
}

(Received 7 May 2021; revised 9 August 2021; accepted 10 August 2021; published 29 October 2021)

\begin{abstract}
The existence of a gapped chiral spin liquid has been recently suggested in the vicinity of the metal-insulator transition of the Hubbard model on the triangular lattice, by intensive density-matrix renormalization group (DMRG) simulations [A. Szasz, J. Motruk, M. P. Zaletel, and J. E. Moore, Phys. Rev. X 10, 021042 (2020)]. Here, we report the results obtained within the variational Monte Carlo technique based upon Jastrow-Slater wave functions, implemented with backflow correlations. As in DMRG calculations, we consider $N$-leg cylinders. For $N=4$ and in the presence of a next-nearest-neighbor hopping, a chiral spin liquid emerges between the metal and the insulator with magnetic quasi-long-range order. Within our approach, the chiral state is gapped and breaks the reflection symmetry. By contrast, for both $N=5$ and 6 , the chiral spin liquid is not the state with the lowest variational energy: in the former case, a nematic spin liquid is found in the entire insulating regime, while for the less frustrated case with $N=6$ the results are very similar to that obtained on two-dimensional clusters [L. F. Tocchio, A. Montorsi, and F. Becca, Phys. Rev. B 102, 115150 (2020)], with an antiferromagnetic phase close to the metal-insulator transition and a nematic spin liquid in the strong-coupling regime.
\end{abstract}

DOI: 10.1103/PhysRevResearch.3.043082

\section{INTRODUCTION}

The quest for spin-liquid states has fascinated the condensed-matter physics community since the first proposal of the resonating-valence bond (RVB) theory by Fazekas and Anderson [1,2]. This approach has been one of the first attempts to describe a Mott insulator without any sort of symmetry breaking, even at zero temperature. In recent years, spin liquids have been reported in an increasing number of materials. Examples are given by Herbertsmithite, which is well described by the Heisenberg model on the kagome lattice [3], organic compounds, such as $\kappa(\mathrm{ET})_{2} \mathrm{Cu}_{2}(\mathrm{CN})_{3}$ and $\mathrm{Me}_{3} \mathrm{EtSb}\left[\mathrm{Pd}(\mathrm{dmit})_{2}\right]_{2}$ [4,5], or transition-metal dichalcogenides, like $1 T-\mathrm{TaS}_{2}$, whose low-temperature behavior could be captured by the Hubbard model on the triangular lattice [6].

An important open question concerns the nature of the insulating phase of the two-dimensional Hubbard model on the triangular lattice at half-filling. Most of the investigations have been concentrated on its strong-coupling regime, where only spin $S=1 / 2$ degrees of freedom are left. Here, spin liquids can be systematically classified, according to the projectivesymmetry group (PSG) theory [7-9]. In particular, one can distinguish between $\mathbb{Z}_{2}$ and $U(1)$ spin liquids, according to the low-energy symmetry of the emerging gauge fields [10]. Starting from the Heisenberg model with nearest-neighbor (NN) superexchange $J$, spin-liquid phases are expected to

Published by the American Physical Society under the terms of the Creative Commons Attribution 4.0 International license. Further distribution of this work must maintain attribution to the author(s) and the published article's title, journal citation, and DOI. be stabilized when including either a next-nearest-neighbor (NNN) coupling $J^{\prime}$ or a four-spin ring-exchange term $K$. The latter can be justified within the fourth-order strong-coupling expansion in $t / U$ and is usually considered for an effective description of density fluctuations close to the Mott transition [11]. As far as the $J-J^{\prime}$ model is concerned, a gapless $U$ (1) spin liquid has been proposed by both variational Monte Carlo (VMC) [12,13] and recent density-matrix renormalization group (DMRG) calculations [14], while older DMRG results suggested the presence of a gapped spin liquid $[15,16]$. In addition, also ring-exchange terms may stabilize a gapless spin liquid (with a spinon Fermi surface), as proposed by earlier VMC studies [17] and confirmed by later DMRG simulations $[18,19]$. Further VMC investigations suggested two other gapless spin-liquid states, neither of them possessing a spinon Fermi surface [20]. However, more recent tensornetwork approaches, implemented from Gutzwiller-projected wave functions, do not support the existence of a gapless spin liquid [21].

Recently, chiral spin liquids attracted much attention because of their similarities with quantum Hall states [22,23]. Interestingly, chiral states may exist not only when the Hamiltonian explicitly breaks time-reversal symmetry (as in the quantum Hall effect) [24], but also as a result of a spontaneous symmetry breaking phenomenon [25]. On the triangular lattice, some evidence of this exotic phase has been obtained by adding a scalar chiral interaction to the Heisenberg Hamiltonian [26,27], or even in a fully symmetric Heisenberg model with superexchange couplings up to the third neighbors [28]. A PSG classification of chiral states is possible, as worked out for the fermionic case for different lattices [29]. In particular, two simple Ansätze can be constructed [30]: The first one (dubbed CSL1) is a $U(1)$ chiral spin liquid, with complex 
hoppings defined on a $2 \times 1$ unit cell and no pairing; the second one (dubbed CSL2) is a Gutzwiller-projected $d+i d$ superconductor.

The situation in the Hubbard model, characterized by a kinetic term $t$ and an on-site Coulomb repulsion $U$, is much less clear. The main difficulty comes from the presence of density fluctuations, whose energy scale, which is related to $U$, is much larger than the typical energy scale of spin fluctuations, i.e., $J=4 t^{2} / U$. Therefore, it is not simple to detect tiny effects related to spin degrees of freedom when density fluctuations are present. In addition, numerical methods like exact diagonalization or DMRG suffer from the fact that the local Hilbert space is doubled with respect to the case of $S=$ $1 / 2$. Nevertheless, this effort is necessary in order to capture density fluctuations that are inevitably present in real materials. The possibility that a spin-liquid phase may exist not only in the strong-coupling regime $U \gg t$, but also close to the metal-insulator transition, has been discussed by different theoretical and numerical approaches in the past [31-37]. The term weak-Mott insulator has been used in this case, namely when a spin liquid intrudes between the weak-coupling metal and the strong-coupling antiferromagnetic insulator [11]. In particular, recent extensive DMRG calculations [38,39] highlighted the possibility for a gapped chiral spin liquid close to the Mott transition. A possible description of such a state has been proposed within a bosonic RVB description [40], as well as within a spin model with the four-spin ring-exchange term [41]. Calculations of Ref. [39] are limited to four-leg cylinders, which highly frustrate the $120^{\circ}$ magnetic pattern, since the corresponding $\mathbf{k}$ vectors are not allowed by the quantization of momenta. Instead, in Ref. [38] also six-leg cylinders have been considered, even if the presence of two almost degenerate momentum sectors at intermediate $U / t$ can make the interpretation of the results not completely trivial. In addition, a recent study at finite temperature, still focusing on four-leg cylinders, highlighted the concomitant presence of chiral correlations and nematic order at finite, but low, temperature and intermediate coupling [42]. Instead, a DMRG investigation on three-leg cylinders suggested that a gapless and nonchiral spin liquid appears close to the Mott transition [43]. A more conventional picture, with a direct transition between a metal and an insulator with magnetic order, has been found in Refs. [44-46]. We would like to remark that the analysis of the insulating phase in the vicinity of the Mott transition is complicated by the significant difference in locating the Mott transition, as observed with the different methods. Finally, the effect of NNN hopping has been addressed in Ref. [47], using the VCA method with few (12) sites, leading to a large spin-liquid region for $t^{\prime} / t>0$, and in a VMC study that suggests always a direct transition between a metal and a magnetic insulator, even with an asymmetry between positive and negative values of $t^{\prime} / t$ [48].

In this work, we present variational Monte Carlo results, based upon Jastrow-Slater wave functions and backflow correlations, for the Hubbard model on the triangular lattice on $N$-leg cylinders, with $N=4,5$, and 6 . The role of the NNN hopping term $t^{\prime}$ is also discussed. On four-leg cylinders, we find a chiral spin liquid on a relatively extended region in the vicinity of the metal-insulator transition, when $t^{\prime} / t<0$. This intermediate phase is gapped, in analogy with DMRG results.
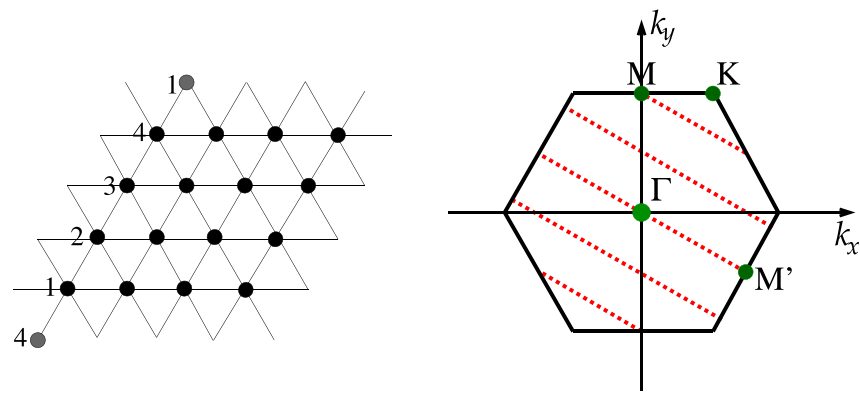

FIG. 1. Left panel: triangular lattice on a cylinder with $L_{2}=4$, with periodic boundary conditions along $\mathbf{a}_{1}$ and $\mathbf{a}_{2}$. This geometry is different from the YC4 geometry of Refs. [38,39]. Right panel: first Brillouin zone of the triangular lattice. The red dashed lines indicate the allowed momenta on a cylinder with $L_{2}=4$.

In addition, a gapless nonchiral state appears at larger values of the Coulomb repulsion. For $t^{\prime} / t \geqslant 0$, the Mott insulator is always nonchiral, with quasi-long-range $120^{\circ}$ magnetic order, even if the chiral spin-liquid state is quite close in energy, at least in the vicinity of the Mott transition. On five- and six-leg cylinders, the chiral spin liquid does not give the best variational energy. For $N=5$, the whole insulating regime is described by a gapless nonchiral spin liquid, while for $N=6$ the phase diagram is similar to the one found in the two-dimensional case [48], with antiferromagnetic order close to the metal-insulator transition and a gapless nonchiral spinliquid phase at strong coupling.

The paper is organized as follows: in Sec. II, we describe the model and the various variational wave functions, as well as the quantities that have been used to obtain the important information; in Sec. III, we present the numerical results; finally, in Sec. IV, we draw our conclusions.

\section{MODEL AND METHOD}

We consider the single-band Hubbard model on the triangular lattice:

$$
\begin{aligned}
\mathcal{H}= & -t \sum_{\langle i, j\rangle, \sigma} c_{i, \sigma}^{\dagger} c_{j, \sigma}-t^{\prime} \sum_{\langle\langle i, j\rangle\rangle, \sigma} c_{i, \sigma}^{\dagger} c_{j, \sigma}+\text { H.c. } \\
& +U \sum_{i} n_{i, \uparrow} n_{i, \downarrow},
\end{aligned}
$$

where $c_{i, \sigma}^{\dagger}\left(c_{i, \sigma}\right)$ creates (destroys) an electron with spin $\sigma$ on site $i$ and $n_{i, \sigma}=c_{i, \sigma}^{\dagger} c_{i, \sigma}$ is the electronic density per spin $\sigma$ on site $i$. The NN and NNN hoppings are denoted as $t$ and $t^{\prime}$, respectively; $U$ is the on-site Coulomb interaction. We define three vectors connecting $\mathrm{NN}$ sites, $\mathbf{a}_{1}=(1,0)$, $\mathbf{a}_{2}=(1 / 2, \sqrt{3} / 2)$, and $\mathbf{a}_{3}=(-1 / 2, \sqrt{3} / 2)$; in addition, we also define three vectors for NNN sites, $\mathbf{b}_{1}=\mathbf{a}_{1}+\mathbf{a}_{2}, \mathbf{b}_{2}=$ $\mathbf{a}_{2}+\mathbf{a}_{3}$, and $\mathbf{b}_{3}=\mathbf{a}_{3}-\mathbf{a}_{1}$. We consider clusters with periodic boundary conditions defined by $\mathbf{T}_{1}=L_{1} \mathbf{a}_{1}$ and $\mathbf{T}_{2}=L_{2} \mathbf{a}_{2}$, in order to have $L=L_{1} \times L_{2}$ sites. We focus on cylinders with four $\left(L_{2}=4\right)$, five $\left(L_{2}=5\right)$, and six $\left(L_{2}=6\right)$ legs; see the case with $L_{2}=4$ in Fig. 1 . Most of the calculations have been done with $L_{1}=30$, which is large enough not to suffer from significant finite-size effects. The half-filled case, where the Mott transition takes place, is considered. In this case, only 
the sign of the ratio $t^{\prime} / t$ is relevant and not the individual signs of $t$ and $t^{\prime}$.

Our numerical results are obtained by means of the VMC method, which is based on the definition of suitable wave functions to approximate the ground-state properties beyond perturbative approaches [49]. In particular, we consider the so-called Jastrow-Slater wave functions that include longrange electron-electron correlations via the Jastrow factor [50,51], on top of an uncorrelated Slater determinant (possibly including electron pairing). In addition, the so-called backflow correlations will be applied to the Slater determinant in order to sizably improve the quality of the variational state $[52,53]$. Thanks to Jastrow and backflow terms, these wave functions can reach a very high degree of accuracy in Hubbard-like models for different regimes of parameters, including frustrated cases [54]. Therefore, they represent a valid tool to investigate strongly correlated systems, competing with state-of-the-art numerical methods, such as DMRG or tensor networks.

Our variational wave function for describing the spinliquid phase is defined as

$$
\left|\Psi_{\mathrm{SL}}\right\rangle=\mathcal{J}_{d}\left|\Phi_{\mathrm{MF}}\right\rangle,
$$

where $\mathcal{J}_{d}$ is the density-density Jastrow factor and $\left|\Phi_{\mathrm{MF}}\right\rangle$ is a state where the orbitals of an auxiliary Hamiltonian are redefined on the basis of the many-body electronic configuration, incorporating virtual hopping processes, via the backflow correlations [52,53].

The density-density Jastrow factor is given by

$$
\mathcal{J}_{d}=\exp \left(-\frac{1}{2} \sum_{i, j} v_{i, j} n_{i} n_{j}\right),
$$

where $n_{i}=\sum_{\sigma} n_{i, \sigma}$ is the electron density on site $i$, and $v_{i, j}$ are pseudopotentials that are optimized for every independent distance $\left|\mathbf{R}_{i}-\mathbf{R}_{j}\right|$. The density-density Jastrow factor allows us to describe a nonmagnetic Mott insulator for a sufficiently singular Jastrow factor $v_{q} \sim 1 / q^{2}\left(v_{q}\right.$ being the Fourier transform of $v_{i, j}$ ) [50,51].

The auxiliary Hamiltonian is then defined as follows:

$$
\mathcal{H}_{\mathrm{MF}}=\sum_{k, \sigma} \xi_{k} c_{k, \sigma}^{\dagger} c_{k, \sigma}+\sum_{k} \Delta_{k} c_{k, \uparrow}^{\dagger} \uparrow_{-k, \downarrow}^{\dagger}+\text { H.c., }
$$

where $\xi_{k}=\tilde{\epsilon}_{k}-\mu$ defines the free-band dispersion (including the chemical potential $\mu$ ) and $\Delta_{k}$ is the singlet pairing amplitude. In our previous work on the two-dimensional lattice [48], we found that the best spin liquid has a nematic character, the hopping terms being given by

$$
\begin{aligned}
\tilde{\epsilon}_{k}= & -2 t\left[\cos \left(\mathbf{k} \cdot \mathbf{a}_{1}\right)+\cos \left(\mathbf{k} \cdot \mathbf{a}_{3}\right)\right]-2 \tilde{\tilde{t}_{d}} \cos \left(\mathbf{k} \cdot \mathbf{a}_{2}\right) \\
& -2 \tilde{t}^{\prime}\left[\cos \left(\mathbf{k} \cdot \mathbf{b}_{1}\right)+\cos \left(\mathbf{k} \cdot \mathbf{b}_{2}\right)+\cos \left(\mathbf{k} \cdot \mathbf{b}_{3}\right)\right] .
\end{aligned}
$$

Instead, the pairing amplitudes are

$$
\Delta_{k}=2 \Delta\left[\cos \left(\mathbf{k} \cdot \mathbf{a}_{1}\right)-\cos \left(\mathbf{k} \cdot \mathbf{a}_{3}\right)\right]+2 \Delta_{d}\left(\cos \mathbf{k} \cdot \mathbf{a}_{2}\right),
$$

which possess a $d$-wave symmetry on the two bonds with hopping $t$. In two dimensions, we found $\tilde{t}_{d} \approx 0$ and $\Delta_{d} \approx 0$, while on cylinders they may assume finite values. Remarkably, this choice (with different couplings along $\mathbf{a}_{2}$ and $\mathbf{a}_{3}$ ) gives the best variational energy also on cylinders, implying an explicit breaking in point-group symmetries.

In addition, we focus on chiral spin-liquid states, which have been claimed to be relevant both in the Heisenberg limit [30] and in the Hubbard model close to the Mott transition $[38,39]$. The CSL2 state is a projected $d+i d$ superconductor characterized by uniform (real) hopping along NN and NNN bonds and a pairing

$$
\Delta_{k}=2 \Delta\left[\cos \left(\mathbf{k} \cdot \mathbf{a}_{1}\right)+\omega \cos \left(\mathbf{k} \cdot \mathbf{a}_{2}\right)+\omega^{2} \cos \left(\mathbf{k} \cdot \mathbf{a}_{3}\right)\right],
$$

where $\omega=e^{2 i \pi / 3}$. Another chiral state (dubbed here CSL3) may be defined by the hopping amplitude of Eq. (5) and a different $d+i d$ pairing structure:

$$
\Delta_{k}=2 \Delta\left[\cos \left(\mathbf{k} \cdot \mathbf{a}_{1}\right)-\cos \left(\mathbf{k} \cdot \mathbf{a}_{3}\right)\right]+2 i \Delta_{d} \cos \left(\mathbf{k} \cdot \mathbf{a}_{2}\right) .
$$

Finally, a chiral spin liquid with $U(1)$ symmetry has been proposed (dubbed CSL1 in Ref. [30]), with magnetic fluxes piercing the elementary plaquettes. In the presence of density fluctuations, this state breaks the translational symmetry and does not give a competitive variational energy. Therefore, in the following, this Ansatz is not reported.

Within the two-dimensional case, antiferromagnetically ordered wave functions represent an important class of states, since a large portion of the phase diagram corresponds to phases that spontaneously break the $\mathrm{SU}(2)$ spin symmetry. Cylinders are quasi-one-dimensional systems, in which a continuous symmetry cannot be broken. Nevertheless, a variational wave function can be still constructed from a magnetically ordered Slater determinant. Then, density and spin correlations may be inserted by Jastrow factors:

$$
\left|\Psi_{\mathrm{AF}}\right\rangle=\mathcal{J}_{s} \mathcal{J}_{d}\left|\Phi_{\mathrm{AF}}\right\rangle
$$

here, $\mathcal{J}_{d}$ is the density-density term of Eq. (3), and $\mathcal{J}_{s}$ is the spin-spin Jastrow factor, which is written in terms of a pseudopotential $u_{i, j}$ that couples the $z$-component of the spin operators on different sites:

$$
\mathcal{J}_{s}=\exp \left(-\frac{1}{2} \sum_{i, j} u_{i, j} S_{i}^{z} S_{j}^{z} .\right.
$$

Finally, $\left|\Phi_{\mathrm{AF}}\right\rangle$ is obtained, after taking into account the backflow corrections, from the following auxiliary Hamiltonian:

$$
\mathcal{H}_{\mathrm{AF}}=\sum_{k, \sigma} \epsilon_{k} c_{k, \sigma}^{\dagger} c_{k, \sigma}+\Delta_{\mathrm{AF}} \sum_{i} \mathbf{M}_{i} \cdot \mathbf{S}_{i},
$$

where $\epsilon_{k}$ is the free dispersion of Eq. (1), $\mathbf{S}_{i}=\left(S_{i}^{x}, S_{i}^{y}, S_{i}^{z}\right)$ is the spin operator at site $i$, and $\mathbf{M}_{i}$ is defined as $\mathbf{M}_{i}=$ $\left[\cos \left(\mathbf{Q} \cdot \mathbf{R}_{i}\right), \sin \left(\mathbf{Q} \cdot \mathbf{R}_{i}\right), 0\right]$, where $\mathbf{Q}$ is the pitch vector. The three-sublattice $120^{\circ}$ order has $\mathbf{Q}=\left(\frac{4 \pi}{3}, 0\right)$ or $\left(\frac{2 \pi}{3}, \frac{2 \pi}{\sqrt{3}}\right)$, while the stripe collinear order with a two-sublattice periodicity has $\mathbf{Q}=\left(0, \frac{2 \pi}{\sqrt{3}}\right)$ or $\mathbf{Q}=\left(\pi, \frac{\pi}{\sqrt{3}}\right)$. On six-leg cylinders, the pitch vector corresponding to the $120^{\circ}$ order is allowed by the quantization of momenta; instead, on four- and five-leg cases it is not allowed, and we take the closest possible momentum. On five legs, also the pitch vector of the stripe collinear order is not allowed.

In general, the effect of the spin-spin Jastrow factor $\mathcal{J}_{s}$ is to reduce the value of magnetic order of the uncorrelated Slater determinant $[55,56]$. In purely one-dimensional systems, the 
presence of a long-range Jastrow factor is able to completely destroy magnetic order, leading to the correct behavior of the spin-spin correlations [57]. On cylinders with a finite number of legs $N$, a residual magnetic order persists, thus giving rise to a spurious wave function that breaks the SU(2) symmetry. Here, we interpret the possibility to stabilize this kind of variational state as the tendency to develop magnetic order in the two-dimensional system. For simplicity, in the following, the Ansatz of Eq. (9) will be denoted by "antiferromagnetic." We remark that, in principle, it would be possible to restore the $\mathrm{SU}(2)$ symmetry by projecting on the $S=0$ subspace [58]. However, this procedure is rather computationally expensive, whenever the computational basis has a definite value of $S^{z}=$ $\sum_{i} S_{i}^{z}$ but not of $S^{2}=\left(\sum_{i} \mathbf{S}_{i}\right)^{2}$. All the pseudopotentials in the Jastrow factors, the parameters in the auxiliary Hamiltonian, as well as the backflow corrections are optimized with the stochastic reconfiguration method [49].

To assess the metallic or insulating nature of the ground state, we can compute the static density-density structure factor:

$$
N(\mathbf{q})=\frac{1}{L} \sum_{i, j}\left\langle n_{i} n_{j}\right\rangle^{i \mathbf{q} \cdot\left(\mathbf{R}_{i}-\mathbf{R}_{j}\right)},
$$

where $\langle\cdots\rangle$ indicates the expectation value over the variational wave function. Indeed, density excitations are gapless when $N(\mathbf{q}) \propto|\mathbf{q}|$ for $|\mathbf{q}| \rightarrow 0$, while a gap is present whenever $N(\mathbf{q}) \propto|\mathbf{q}|^{2}$ for $|\mathbf{q}| \rightarrow 0 \quad[53,59]$. Analogously, the presence of a spin gap can be checked by looking at the small- $q$ behavior of the static spin-spin correlations [60]:

$$
S(\mathbf{q})=\frac{1}{L} \sum_{i, j}\left\langle S_{i}^{z} S_{j}^{z}\right\rangle^{i \mathbf{q} \cdot\left(\mathbf{R}_{i}-\mathbf{R}_{j}\right)} .
$$

\section{RESULTS}

Here, we discuss the results for the variational energy of different states on the four-leg cylinder geometry. Let us start from the case with $t^{\prime} / t=0$; see Fig. 2 (upper panel). In this case, the Mott transition occurs between $U / t=9$ and 9.5, as extracted from the low- $q$ behavior of the density-density correlations; see Fig. 3 (upper panel). In the small- $U$ regime, the pairing terms $\Delta_{k}$ [in the spin-liquid Ansätze of Eq. (2)] or the antiferromagnetic parameter $\Delta_{\mathrm{AF}}$ [in the magnetic wave function of Eq. (9)] are very small and there is a tiny difference among all different variational states. This fact indicates that the conducting phase is a standard metal, with neither magnetic nor superconducting order. Instead, in the insulating phase, the optimal wave function is the antiferromagnetic one with the pitch vector corresponding to approximately $120^{\circ}$ order, i.e., $\mathbf{Q}=\left(\frac{2 \pi}{3}, \frac{7 \pi}{3 \sqrt{3}}\right)$. The overall situation is not much different from what has been obtained, within the same approach, in the two-dimensional limit [48] (except the fact that in the latter case, a true antiferromagnetic order settles down). We also remark that the energy gain of the antiferromagnetic state with respect to the spin-liquid one is smaller on four legs than in two dimensions.

Then, a large spin-liquid region appears immediately above the Mott transition, by including a finite NNN hopping $t^{\prime} / t=$ -0.3 ; see Fig. 2 (middle panel). Here, the metal-insulator transition takes place at $U / t=11.5 \pm 0.5$; see Fig. 3 (middle
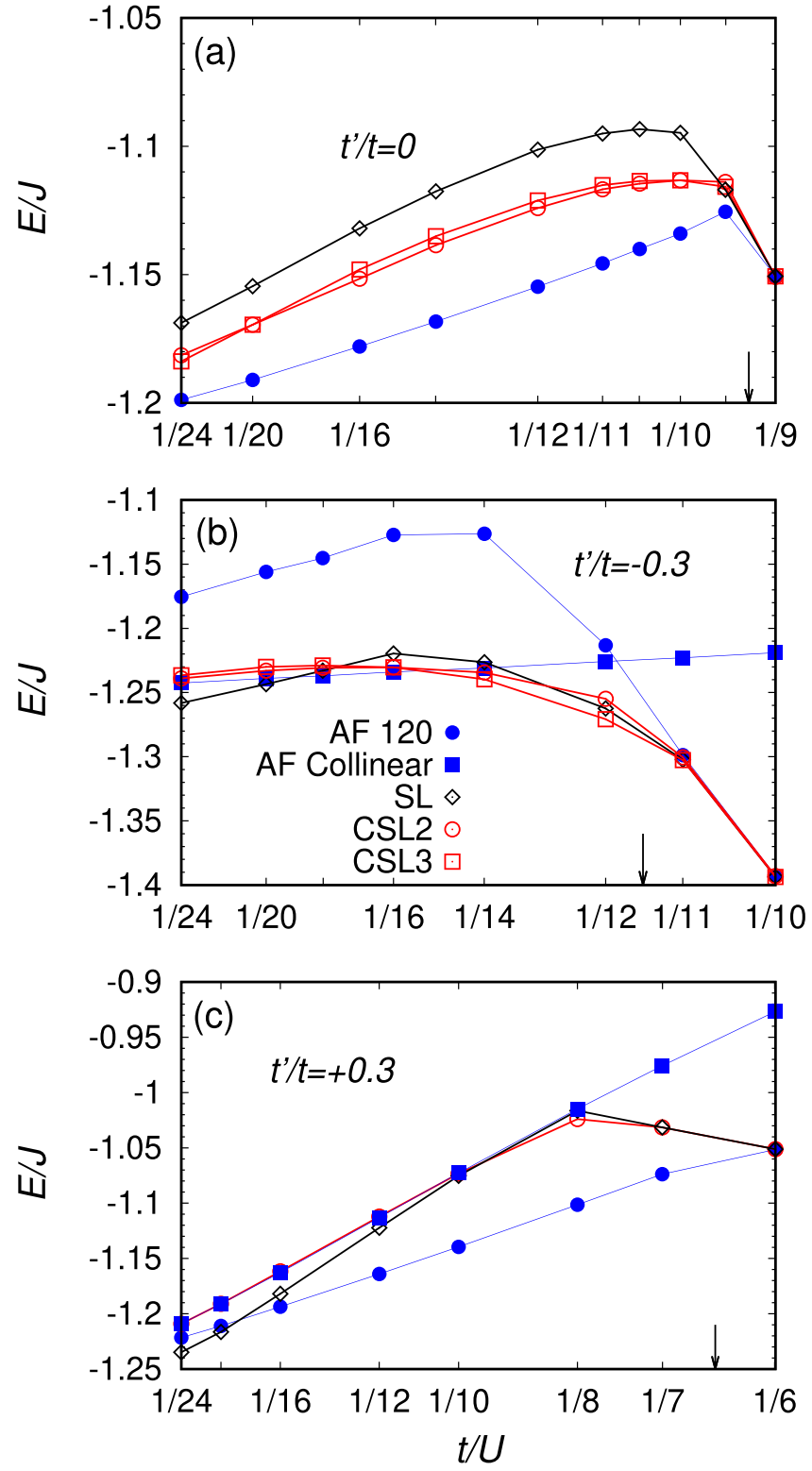

FIG. 2. Energy (per site) in units of $J=4 t^{2} / U$, as a function of $t / U$ on the four-leg cylinder for $t^{\prime} / t=0$ (upper panel), $t^{\prime} / t=$ -0.3 (middle panel), and $t^{\prime} / t=+0.3$ (lower panel). Data are shown for different trial wave functions: the antiferromagnetic state with $\mathbf{Q}=\left(\frac{2 \pi}{3}, \frac{7 \pi}{3 \sqrt{3}}\right)$ (blue full circles), the one with $\mathbf{Q}=\left(0, \frac{2 \pi}{\sqrt{3}}\right)$ (blue full squares), the spin liquid (SL) with hopping in Eq. (5) and pairing in Eq. (6) (black empty diamonds), the CSL2 with uniform hopping and pairing given by Eq. (7) (red empty circles), and the CSL3 defined by Eqs. (5) and (8) (red empty squares). Black arrows denote the metal-insulator transitions. Data are shown for an $L=30 \times 4$ lattice size. Error bars are smaller than the symbol size.

panel). The best variational state, between $U / t=12$ and 16 , is given by the CSL3, even though the other spin-liquid states are very close in energy. By increasing the ratio $U / t$, the ground state passes through an intermediate phase where the best variational state is the antiferromagnetic one (with collinear order), before entering a further (strong-coupling) spin-liquid region that has no chiral features, in analogy with the results previously obtained in two dimensions [48]. In Fig. 2, we do 

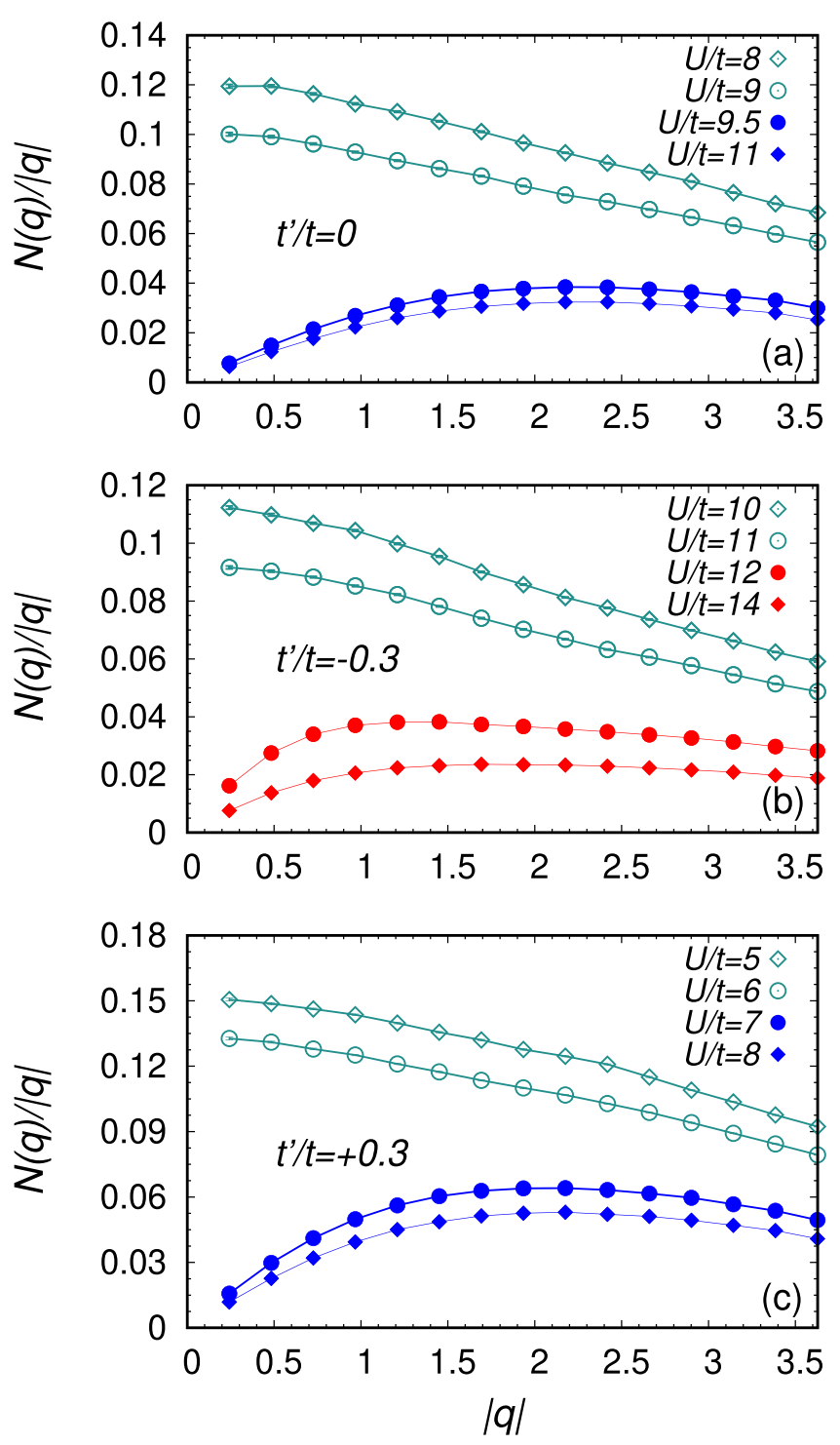

FIG. 3. Static density-density structure factor $N(\mathbf{q})$, divided by $|\mathbf{q}|$, computed for the lowest-energy states at different values of $U / t$. Results for $t^{\prime} / t=0$ (upper panel), $t^{\prime} / t=-0.3$ (middle panel), and $t^{\prime} / t=+0.3$ (lower panel) are shown on a four-leg cylinder with $L=30 \times 4$, along the line connecting $\Gamma=(0,0)$ to $M^{\prime}=\left(\pi,-\frac{\pi}{\sqrt{3}}\right)$. Error bars are smaller than the symbol size.

not report the flux phase CSL1, since its variational energy is always significantly higher than the other states. For example, at $U / t=12$, we have $E / J=-0.8907$ (3) for $t^{\prime}=0$ and $E / J=-0.9294(3)$ for $t^{\prime} / t=-0.3$. The situation is radically different by taking the opposite sign of the hopping amplitudes, i.e., $t^{\prime} / t=+0.3$; see Fig. 2 (lower panel). The Mott transition lowers down to $U / t=6.5 \pm 0.5$ [see Fig. 3 (lower panel)], with the insulating state being approximated by the antiferromagnetic state, with pitch vector $\mathbf{Q}=\left(\frac{2 \pi}{3}, \frac{7 \pi}{3 \sqrt{3}}\right)$, up to $U / t \approx 20$. For larger values of the electron-electron repulsion, a nonchiral spin-liquid state emerges. Note that the energies reported for the antiferromagnetic state with collinear order below the Mott transition correspond to a local minimum with insulating features.

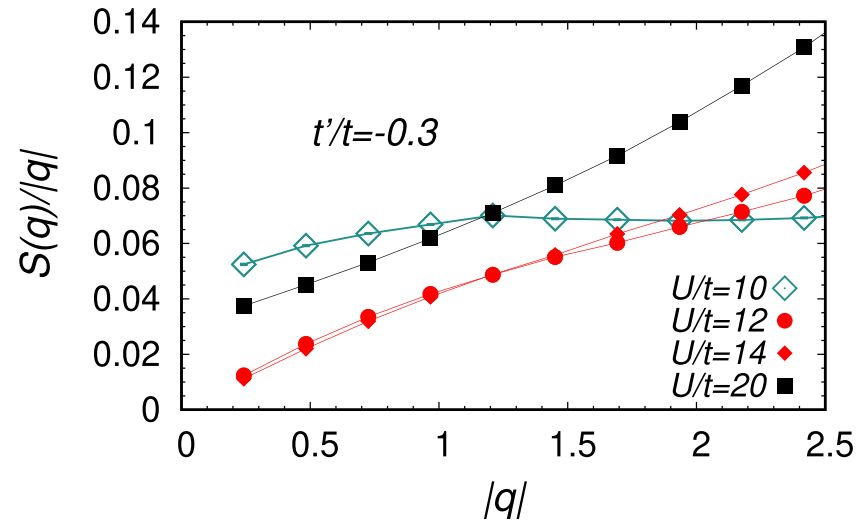

FIG. 4. Static spin-spin structure factor $S(\mathbf{q})$, divided by $|\mathbf{q}|$, computed for the lowest-energy states at different values of $U / t$, for $t^{\prime} / t=-0.3$. Data are shown on a four-leg cylinder with $L=30 \times 4$, in the metallic region (green empty diamonds), for the chiral spin liquid state CSL3 (red full points) and for the large- $U$ spin liquid (black full squares). The $\mathbf{q}$ points are located along the line connecting $\Gamma=(0,0)$ to $M^{\prime}=\left(\pi,-\frac{\pi}{\sqrt{3}}\right)$. Error bars are smaller than the symbol size.

To determine the nature of the chiral spin-liquid state, we analyze the spin-spin correlations by computing the spin-spin structure factor of Eq. (13). In Fig. 4, we report calculations for $t^{\prime} / t=-0.3$ and values of $U / t$ across the Mott transition. The main result is that the chiral spin liquid, realized close to the Mott transition, has a spin gap, since $S(\mathbf{q}) \propto|\mathbf{q}|^{2}$ for small values of the momentum q. This is in agreement with recent DMRG studies [38,39]. We remark that this feature is solid, since it is also shared by the other two spin-liquid states with nearby energies, i.e., the CSL2 and the nonchiral one parametrized by Eqs. (5) and (6). On the contrary, the large- $U$ state is gapless. In this regime the optimal parameters $\tilde{t}_{d} \approx 0$ and $\Delta_{d} \approx 0$ lead to a gapless spectrum in the auxiliary Hamiltonian (4), thus indicating that the nature of the unprojected state is not changed when including the Jastrow factor.

The optimal chiral spin liquid (close to the Mott transition), as well as the nonchiral one (in the strong-coupling regime), are very anisotropic, as shown by computing the nearestneighbor spin-spin correlations $D_{j}=\left\langle S_{\mathbf{R}_{i}}^{z} S_{\mathbf{R}_{i}+\mathbf{a}_{j}}^{z}\right\rangle$, with $j=$ $1,2,3$. For example, for $t^{\prime} / t=-0.3$ and $U / t=12$, the CSL3 state has $D_{1}=D_{3}=-0.029(1)$ and $D_{2}=-0.069(1)$. For $U / t=20$, the nonchiral spin liquid has $D_{1}=D_{3}=$ $-0.101(1)$ and $D_{2}=+0.041(1)$. Within the error bar, these results are the same from $L=18 \times 4$ to $L=30 \times 4$. As discussed in Sec. II, this anisotropy follows directly the parametrization of the spin-liquid state; see Eqs. (5) and (6) for the nonchiral Ansatz and Eqs. (5) and (8) for the CSL3.

Then, we show the stability of the chiral spin liquid when going from $N=4$ to 6 . Results are shown in Fig. 5, together with the ones for a truly two-dimensional cluster (with $L=$ $18 \times 18$ sites), which has already been discussed in our previous work [48]. On a two-dimensional cluster, the CSL3 state is a local minimum, with energy higher than the other states. Instead, on six-leg cylinders, the CSL3 state is not reported, since, upon optimization, it converges to the nonchiral state. The most important fact is that no chiral phases are present 

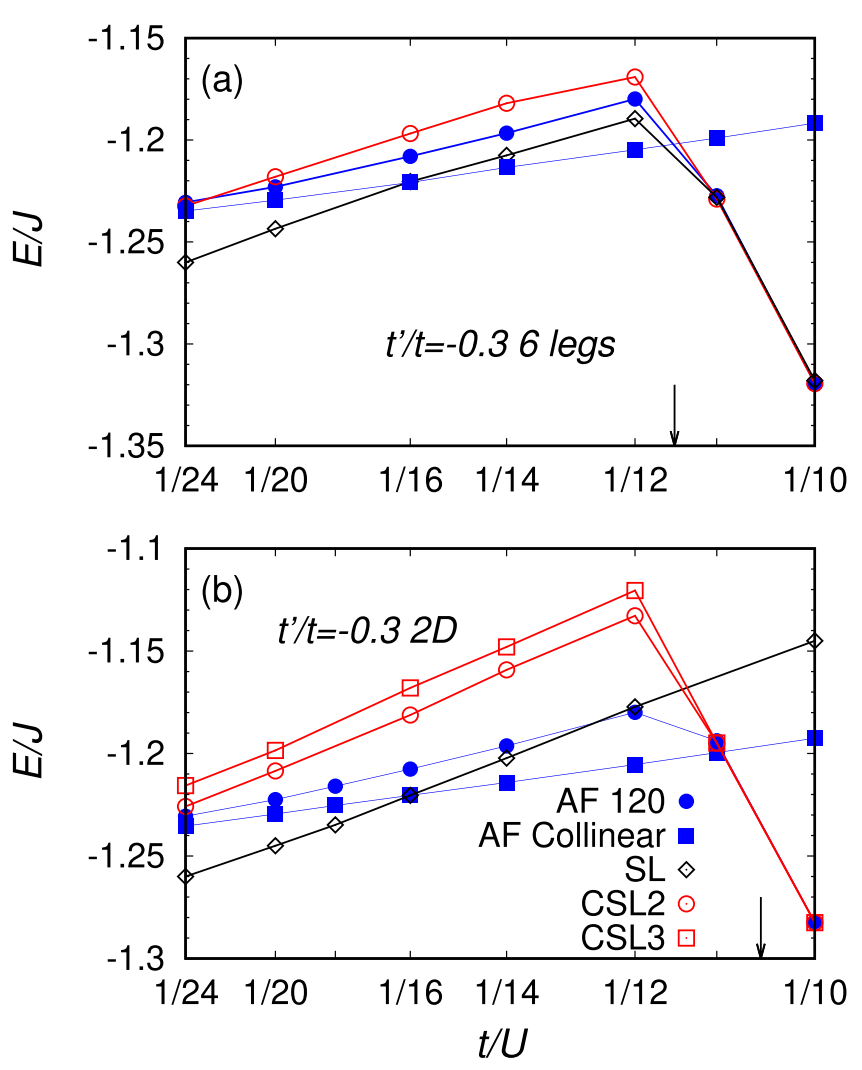

FIG. 5. Energy (per site) in units of $J=4 t^{2} / U$, as a function of $t / U$, for $t^{\prime} / t=-0.3$ on the six-leg cylinder with $L=30 \times 6$ (upper panel). The results for the two-dimensional cluster with $L=18 \times 18$ are also reported for comparison (lower panel). Data are shown for different trial wave functions: The antiferromagnetic state with $120^{\circ}$ magnetic order (blue full circles), the state with collinear order (blue full squares), the spin liquid with hopping in Eq. (5) and pairing in Eq. (6) (black empty diamonds), the CSL2 with uniform hopping and pairing given by Eq. (7) (red empty circles), and the CSL3 defined by Eqs. (5) and (8) (red empty squares). Black arrows denote the metal-insulator transitions. Error bars are smaller than the symbol size.

in the insulating region close to the metal-insulator transition (which, for $N=6$, appears between $U / t=11$ and 12). Here, the insulating phase is either an antiferromagnet with collinear order, in the vicinity of the Mott transition, or a gapless nonchiral spin liquid, in the strong-coupling regime. Note that, also in this case, the antiferromagnetic state with collinear order becomes a local minimum below the Mott transition. The reason for the stabilization of the chiral state on four-leg cylinders comes from its remarkable energy gain when going from $N=6$ (or equivalently two dimensions) to $N=4$; by contrast, the variational energies of the antiferromagnetic state do not change much when varying $N$. Overall, the resulting phase diagram for $N=6$ is qualitatively similar to the one obtained in two dimensions. Therefore, within our approach, the chiral spin liquid exists only for particular values of $N$, like on four-leg cylinders.

Finally, we have also considered cylinders with an odd number of legs, i.e., with $L_{2}=5$. This is a particularly frustrating case, since both $120^{\circ}$ and stripe collinear magnetic correlations are not allowed by the quantization of transverse

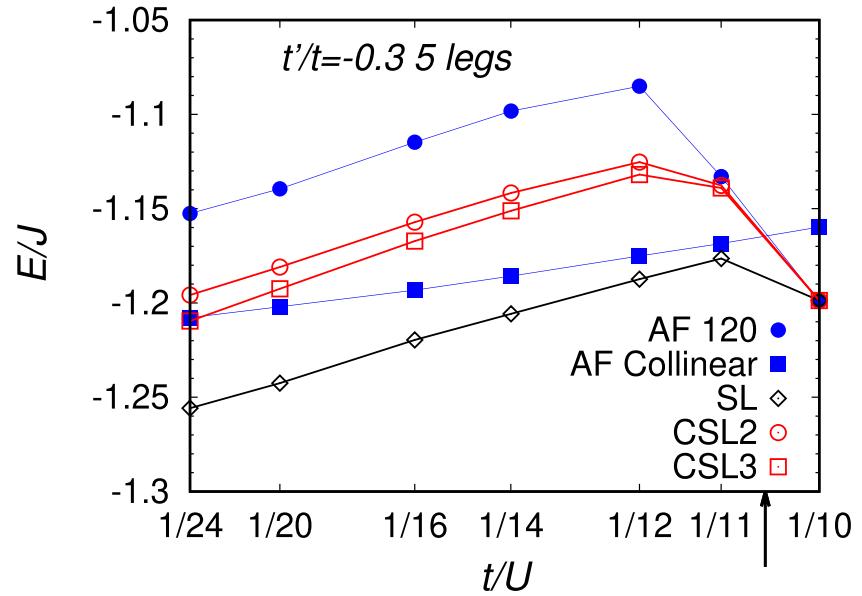

FIG. 6. Energy (per site) in units of $J=4 t^{2} / U$, as a function of $t / U$, for $t^{\prime} / t=-0.3$ on the five-leg cylinder with $L=30 \times 5$. Data are shown for different trial wave functions: The antiferromagnetic state with $\mathbf{Q}=\left(\frac{2 \pi}{3}, \frac{26 \pi}{15 \sqrt{3}}\right)$ (blue full circles), the antiferromagnetic state with $\mathbf{Q}=\left(\pi, \frac{3 \pi}{5 \sqrt{3}}\right)$ (blue full squares), the spin liquid with hopping in Eq. (5) and pairing in Eq. (6) (black empty diamonds), the CSL2 with uniform hopping and pairing given by Eq. (7) (red empty circles), and the CSL3 defined by Eqs. (5) and (8) (red empty squares). The black arrow denotes the metal-insulator transition. Error bars are smaller than the symbol size.

momenta. Results for the energies of the different variational states are reported in Fig. 6. The Mott transition is determined also in this case by looking at the static structure factor of Eq. (12). In this case, the insulator is always a gapless spin liquid, with no chiral features. Indeed, the best variational state is the one defined by Eqs. (5) and (6), with optimal variational parameters $\Delta_{d} \approx 0$ and $\tilde{t}_{d} \approx 0$, which is the same as the large$U$ spin liquid reported on the four-leg case. The two magnetic states are now both disfavored because of the five-leg geometry and they are approximated by the pitch vectors $\mathbf{Q}=$ $\left(\pi, \frac{3 \pi}{5 \sqrt{3}}\right)$ (for the stripe collinear order) and $\mathbf{Q}=\left(\frac{2 \pi}{3}, \frac{26 \pi}{15 \sqrt{3}}\right)$ (for the $120^{\circ}$ order). The two chiral states (CSL2 and CSL3) have also higher energies with respect to the nonchiral one. Our finding is in agreement with that reported by DMRG in Ref. [38], where no chiral features are observed on the five-leg cylinder when using periodic boundary conditions.

\section{CONCLUSIONS}

In summary, we have studied the Hubbard model on cylinders with a triangular lattice geometry by means of the VMC approach. Both a NN hopping $t$ and a NNN hopping $t^{\prime}$ are considered in the model. First, we focused on the four-leg case, with different values of the ratio $t^{\prime} / t$. For $t^{\prime} / t<0$, a spin liquid is stabilized in the vicinity of the Mott transition. This state is a gapped chiral spin liquid that also breaks the point-group symmetry. At larger values of $U / t$, a further gapless spin liquid appears. For $t^{\prime}=0$, the insulating region is always antiferromagnetic (with approximately $120^{\circ}$ order), while for $t^{\prime} / t>0$ we observe a gapless spin liquid in the strong-coupling regime. However, the chiral spin liquid disappears on cylinders with five and six legs, as well as in the truly two-dimensional case. In these cases, a gapless spin liquid 


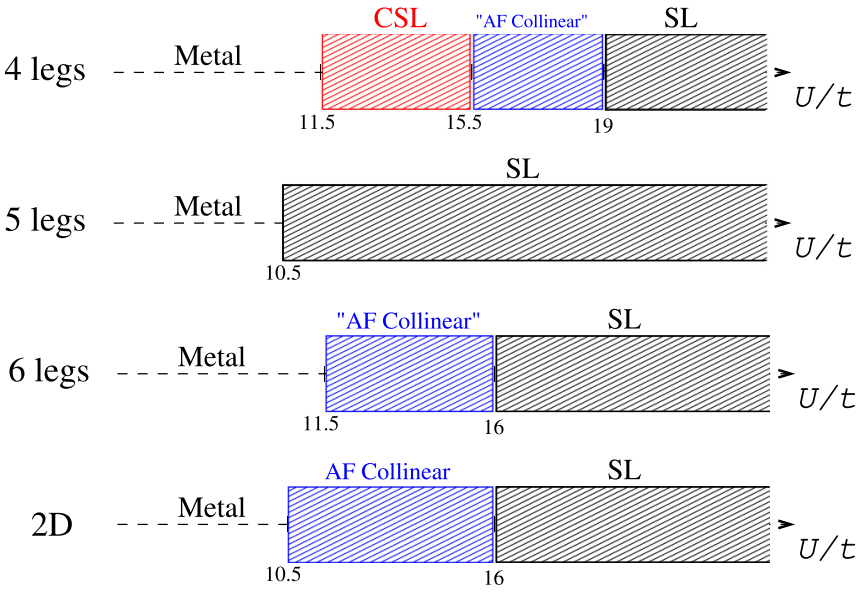

FIG. 7. Schematic phase diagram of the triangular lattice at $t^{\prime} / t=-0.3$ as a function of $U / t$. Four different lattices are considered, with four, five, and six legs, as well as a two-dimensional cluster. The symbol "SL" denotes a gapless spin liquid, while the symbol "CSL" denotes a chiral gapped spin liquid. The antiferromagnetic collinear order on cylindrical geometries is denoted in quotation marks, since no true long-range magnetic order can occur.

survives in the large- $U$ region. These results are summarized in Fig. 7.

Our calculations convey two main messages: On one side, the spin liquid that we obtain on the four-leg cylinder, close to the Mott transition, is chiral and spin gapped, in agreement with recent DMRG calculations [38,39]. In addition, the best chiral state breaks the reflection symmetry, as also suggested in the finite-temperature tensor-network method of Ref. [42]. Nevertheless, within variational Monte Carlo, an additional NNN hopping is necessary to stabilize the chiral state. On the other side, our results suggest that a chiral spin liquid exists only in particular geometries (e.g., the four-leg cylinder). Instead, on cylinders with five and six legs (as well as in two dimensions), the chiral spin liquid either is not stable upon optimization or has a variational energy that is quite higher than the optimal state. Finally, we observe that chiral flux phases (defined on the $2 \times 1$ unit cell) have a variational energy that is not competitive with other wave functions. As with all variational calculations, our results suffer from an intrinsic bias, given by the choice of the variational Ansatz; still, the Jastrow-Slater state possesses a great deal of flexibility, being able to describe a wide variety of different phases, including quantum spin liquids, with or without chiral order. The fact that we do not observe a chiral spin liquid in five- and six-leg cylinders and in two-dimensional clusters suggests that either this state is not present or it cannot be represented by the Ansätze that have been considered here.

\section{ACKNOWLEDGMENT}

We thank F. Ferrari for insightful discussions.
[1] P. W. Anderson, Resonating valence bonds: A new kind of insulator? Mater. Res. Bull. 8, 153 (1973).

[2] P. Fazekas and P. W. Anderson, On the ground state properties of the anisotropic triangular antiferromagnet, Philos. Mag. 30, 423 (1974).

[3] M. R. Norman, Colloquium: Herbertsmithite and the search for the quantum spin liquid, Rev. Mod. Phys. 88, 041002 (2016).

[4] K. Kanoda and R. Kato, Mott physics in organic conductors with triangular lattices, Annu. Rev. Condens. Matter Phys. 2, 167 (2011).

[5] B. J. Powell and R. H. McKenzie, Quantum frustration in organic Mott insulators: from spin liquids to unconventional superconductors, Rep. Prog. Phys. 74, 056501 (2011).

[6] K. T. Law and P. A. Lee, $1 \mathrm{~T}-\mathrm{TaS}_{2}$ as a quantum spin liquid, Proc. Natl. Acad. Sci. (USA) 114, 6996 (2017).

[7] Y. Zhou and X.-G. Wen, Quantum orders and spin liquids in $\mathrm{Cs}_{2} \mathrm{CuCl}_{4}$, arXiv:cond-mat/0210662.

[8] F. Wang and A. Vishwanath, Spin-liquid states on the triangular and kagomé lattices: A projective-symmetry-group analysis of Schwinger boson states, Phys. Rev. B 74, 174423 (2006).

[9] Y.-M. Lu, Symmetric $Z_{2}$ spin liquids and their neighboring phases on triangular lattice, Phys. Rev. B 93, 165113 (2016).

[10] X.-G. Wen, Quantum orders and symmetric spin liquids, Phys. Rev. B 65, 165113 (2002).

[11] T. Grover, N. Trivedi, T. Senthil, and P. A. Lee, Weak Mott insulators on the triangular lattice: Possibility of a gapless nematic quantum spin liquid, Phys. Rev. B 81, 245121 (2010).
[12] R. Kaneko, S. Morita, and M. Imada, Gapless spin-liquid phase in an extended spin 1/2 triangular Heisenberg model, J. Phys. Soc. Jpn. 83, 093707 (2014).

[13] Y. Iqbal, W.-J. Hu, R. Thomale, D. Poilblanc, and F. Becca, Spin liquid nature in the Heisenberg $J_{1}-J_{2}$ triangular antiferromagnet, Phys. Rev. B 93, 144411 (2016).

[14] S. Hu, W. Zhu, S. Eggert, and Y.-C. He, Dirac Spin Liquid on the Spin-1/2 Triangular Heisenberg Antiferromagnet, Phys. Rev. Lett. 123, 207203 (2019).

[15] Z. Zhu and S. R. White, Spin liquid phase of the $S=\frac{1}{2} J_{1}-J_{2}$ Heisenberg model on the triangular lattice, Phys. Rev. B 92, 041105(R) (2015).

[16] W.-J. Hu, S.-S. Gong, W. Zhu, and D. N. Sheng, Competing spin-liquid states in the spin- $\frac{1}{2}$ Heisenberg model on the triangular lattice, Phys. Rev. B 92, 140403(R) (2015).

[17] O. I. Motrunich, Variational study of triangular lattice spin-1/2 model with ring exchanges and spin liquid state in $\kappa-(\mathrm{ET})_{2} \mathrm{Cu}_{2}(\mathrm{CN})_{3}$, Phys. Rev. B 72, 045105 (2005).

[18] M. S. Block, D. N. Sheng, O. I. Motrunich, and M. P. A. Fisher, Spin Bose-Metal and Valence Bond Solid Phases in a Spin-1/2 Model With Ring Exchanges on a Four-Leg Triangular Ladder, Phys. Rev. Lett. 106, 157202 (2011).

[19] W.-Y. Hu, X. Y. Xu, G. Chen, K. T. Law, and P. A. Lee, Spinon Fermi Surface in a Cluster Mott Insulator Model on a Triangular Lattice and Possible Application to $1 \mathrm{~T}-\mathrm{TaS}_{2}$, Phys. Rev. Lett. 121, 046401 (2018). 
[20] R. V. Mishmash, J. R. Garrison, S. Bieri, and C. Xu, Theory of a Competitive Spin Liquid State for Weak Mott Insulators on the Triangular Lattice, Phys. Rev. Lett. 111, 157203 (2013).

[21] A. M. Aghaei, B. Bauer, K. Shtengel, and R. V. Mishmash, Efficient matrix-product-state preparation of highly entangled trial states: Weak Mott insulators on the triangular lattice revisited, arXiv:2009.12435.

[22] V. Kalmeyer and R. B. Laughlin, Equivalence of the Resonating-Valence-Bond and Fractional Quantum Hall States, Phys. Rev. Lett. 59, 2095 (1987); Theory of the spin liquid state of the Heisenberg antiferromagnet, Phys. Rev. B 39, 11879 (1989).

[23] X.-G. Wen, F. Wilczek, and A. Zee, Chiral spin states and superconductivity, Phys. Rev. B 39, 11413 (1989).

[24] B. Bauer, L. Cincio, B. P. Keller, M. Dolfi, G. Vidal, S. Trebst, and A. W. W. Ludvig, Chiral spin liquid and emergent anyons in a Kagome lattice Mott insulator, Nat. Commun. 5, 5137 (2014).

[25] Y.-C. He, D. N. Sheng, Y. Chen, Chiral Spin Liquid in a Frustrated Anisotropic Kagome Heisenberg Model, Phys. Rev. Lett. 112, 137202 (2014)

[26] W.-J. Hu, S.-S. Gong, and D. N. Sheng, Variational Monte Carlo study of chiral spin liquid in quantum antiferromagnet on the triangular lattice, Phys. Rev. B 94, 075131 (2016).

[27] A. Wietek and A. M. Läuchli, Chiral spin liquid and quantum criticality in extended $S=\frac{1}{2}$ Heisenberg models on the triangular lattice, Phys. Rev. B 95, 035141 (2017).

[28] S.-S. Gong, W. Zheng, M. Lee, Y.-M. Lu, and D. N. Sheng, Chiral spin liquid with spinon Fermi surfaces in the spin- $\frac{1}{2}$ triangular Heisenberg model, Phys. Rev. B 100, 241111(R) (2019).

[29] S. Bieri, C. Lhuillier, and L. Messio, Projective symmetry group classification of chiral spin liquids, Phys. Rev. B 93, 094437 (2016).

[30] X.-Y. Song, A. Vishwanath, and Y.-H. Zhang, Doping the chiral spin liquid: Topological superconductor or chiral metal, Phys. Rev. B 103, 165138 (2021).

[31] P. Sahebsara and D. Sénéchal, Hubbard Model on the Triangular Lattice: Spiral Order and Spin Liquid, Phys. Rev. Lett. 100, 136402 (2008).

[32] A. Yamada, Magnetic properties and Mott transition in the Hubbard model on the anisotropic triangular lattice, Phys. Rev. B 89, 195108 (2014).

[33] M. Laubach, R. Thomale, C. Platt, W. Hanke, and G. Li, Phase diagram of the Hubbard model on the anisotropic triangular lattice, Phys. Rev. B 91, 245125 (2015).

[34] T. Yoshioka, A. Koga, and N. Kawakami, Quantum Phase Transitions in the Hubbard Model on a Triangular Lattice, Phys. Rev. Lett. 103, 036401 (2009).

[35] H.-Y. Yang, A. M. Läuchli, F. Mila, and K. P. Schmidt, Effective Spin Model for the Spin-Liquid Phase of the Hubbard Model on the Triangular Lattice, Phys. Rev. Lett. 105, 267204 (2010).

[36] A. E. Antipov, A. N. Rubtsov, M. I. Katsnelson, and A. I. Lichtenstein, Electron energy spectrum of the spin-liquid state in a frustrated Hubbard model, Phys. Rev. B 83, 115126 (2011).

[37] T. Shirakawa, T. Tohyama, J. Kokalj, S. Sota, and S. Yunoki, Ground-state phase diagram of the triangular lattice Hubbard model by the density-matrix renormalization group method, Phys. Rev. B 96, 205130 (2017).
[38] A. Szasz, J. Motruk, M. P. Zaletel, and J. E. Moore, Chiral Spin Liquid Phase of the Triangular Lattice Hubbard Model: A Density Matrix Renormalization Group Study, Phys. Rev. X 10, 021042 (2020).

[39] B.-B. Chen, Z. Chen, S.-S. Gong, D. N. Sheng, W. Li, and A. Weichselbaum, Quantum spin liquid with emergent chiral order in the triangular-lattice Hubbard model, arXiv:2102.05560.

[40] Q. Zhang and T. Li, Bosonic resonating valence bond theory of the possible chiral spin-liquid state in the triangular-lattice Hubbard model, Phys. Rev. B 104, 075103 (2021).

[41] T. Cookmeyer, J. Motruk, and J. E. Moore, Four-Spin Terms and the Origin of the Chiral Spin Liquid in Mott Insulators on the Triangular Lattice, Phys. Rev. Lett. 127, 087201 (2021).

[42] A. Wietek, R. Rossi, F. Šimkovic IV, M. Klett, P. Hansmann, M. Ferrero, E. Miles Stoudenmire, T. Schäfer, and A. Georges, Mott insulating states with competing orders in the triangular lattice Hubbard model, Phys. Rev. X 11, 041013 (2021).

[43] C. Peng, Y.-F. Jiang, Y. Wang, H.-C. Jiang, Gapless spin liquid and pair density wave of the Hubbard model on three-leg triangular cylinders, arXiv:2103.07998.

[44] H. Lee, G. Li, and H. Monien, Hubbard model on the triangular lattice using dynamical cluster approximation and dual fermion methods, Phys. Rev. B 78, 205117 (2008).

[45] T. Watanabe, H. Yokoyama, Y. Tanaka, and J. Inoue, Predominant magnetic states in the Hubbard model on anisotropic triangular lattices, Phys. Rev. B 77, 214505 (2008).

[46] L. F. Tocchio, H. Feldner, F. Becca, R. Valentí, and C. Gros, Spin-liquid versus spiral-order phases in the anisotropic triangular lattice, Phys. Rev. B 87, 035143 (2013).

[47] K. Misumi, T. Kaneko, and Y. Ohta, Mott transition and magnetism of the triangular-lattice Hubbard model with nextnearest-neighbor hopping, Phys. Rev. B 95, 075124 (2017).

[48] L. F. Tocchio, A. Montorsi, and F. Becca, Magnetic and spinliquid phases in the frustrated $t-t^{\prime}$ Hubbard model on the triangular lattice, Phys. Rev. B 102, 115150 (2020).

[49] F. Becca and S. Sorella, Quantum Monte Carlo approaches for correlated systems (Cambridge University Press, Cambridge, 2017).

[50] M. Capello, F. Becca, M. Fabrizio, S. Sorella, and E. Tosatti, Variational Description of Mott Insulators, Phys. Rev. Lett. 94, 026406 (2005).

[51] M. Capello, F. Becca, S. Yunoki, and S. Sorella, Unconventional metal-insulator transition in two dimensions, Phys. Rev. B 73, 245116 (2006).

[52] L. F. Tocchio, F. Becca, A. Parola, and S. Sorella, Role of backflow correlations for the nonmagnetic phase of the $t-t^{\prime}$ Hubbard model, Phys. Rev. B 78, 041101(R) (2008).

[53] L. F. Tocchio, F. Becca, and C. Gros, Backflow correlations in the Hubbard model: An efficient tool for the study of the metal-insulator transition and the large- $U$ limit, Phys. Rev. B 83, 195138 (2011)

[54] J. P. F. LeBlanc, A. E. Antipov, F. Becca, I. W. Bulik, G. Kin-Lic Chan, C.-M. Chung, Y. Deng, M. Ferrero, T. M. Henderson, C. A. Jimenez-Hoyos, E. Kozik, W. Liu, A. J. Millis, N. V. Prokofev, M. Qin, G. E. Scuseria, H. Shi, B. V. Svistunov, L. F. Tocchio, I. S. Tupitsyn, S. R. White et al., Solutions of the Two-Dimensional Hubbard Model: Benchmarks and Results from a Wide Range of Numerical Algorithms, Phys. Rev. X 5, 041041 (2015) 
[55] E. Manousakis, The spin- $\frac{1}{2}$ Heisenberg antiferromagnet on a square lattice and its application to the cuprous oxides, Rev. Mod. Phys. 63, 1 (1991).

[56] F. Becca, M. Capone, and S. Sorella, Spatially homogeneous ground state of the two-dimensional Hubbard model, Phys. Rev. B 62, 12700 (2000).

[57] F. Franjic and S. Sorella, Spin-wave wave function for quantum spin models, Prog. Theor. Phys. 97, 399 (1997).
[58] T. Mizusaki and M. Imada, Quantum-number projection in the path-integral renormalization group method, Phys. Rev. B 69. 125110 (2004).

[59] R. P. Feynman, Atomic theory of the two-fluid model of liquid helium, Phys. Rev. 94, 262 (1954).

[60] L. F. Tocchio, F. Becca, and A. Montorsi, Superconductivity in the Hubbard model: A hidden-order diagnostics from the Luther-Emery phase on ladders, SciPost Phys. 6, 018 (2019). 